\title{
Usability Evaluation of a PDA Interface for Exploration Mobile Robots
}

\author{
Alberto Valero-Gomez* Paloma de la Puente** \\ *Universidad Carlos III de Madrid, Spain(e-mail: alberto.valero@uc3m.es). \\ ** Centro de Automatica y Robotica CSIC-UPM, Madrid, Spain (e-mail: \\ paloma.delapuente@upm.es)
}

\begin{abstract}
In this paper we are presenting a PDA interface for operating a mobile robot remotely. The interface is designed for exploration robotics, in which a roving operator, equipped with a hand-held device can partly share with the robot the scenario to be explored. Based on user evaluation we improved the first prototype and provided some guidelines for PDA interfaces design. The second protoype with the contributed improvements is also presented.
\end{abstract}

Keywords: Teleoperation, human factors, human machine interface, mobile robots, computer interfaces, computer experiments

\section{INTRODUCTION}

Exploration robotics involves the deployment of autonomous robots that work in complex, real-world scenarios. In real missions, users are not likely to be experts in robotics, but they will have considerable domain knowledge concerning the exploration task, the environment, the possible hazards, etc. Even if the robot were able to work in complete autonomy, its humanpartner would still have to retrieve and understand the information collected by the sensors. Furthermore, in many situations the robot may be unable to finalize its task autonomously, with the result that the operator should understand the status of the robot, its surroundings and its capabilities, in order to command the robot with a competence that counterbalances the limitations of its autonomy. Human and robot must collaborate in order to accomplish the given task.

In the last several years, there has been a surge of great interest in human-robot interface design. Adams (2002) article "Critical Considerations for Human-Robot Interface Development," set researchers to work on how to fill the gap between human factors engineering and robotic research. Drury, Yanco, Scholtz and Adams herself, as well as their collaborators, have applied the knowledge thus gained to the specific field of exploration robotics in a number of publications: Scholtz et al. (2004); Drury et al. (2004b); Yanco and Drury (2007); Drury et al. (2004a); Humphrey et al. (2007); Kaymaz-Keskinpala and Adams (2004). These works have resulted in a set of guidelines for Human-Robot Interface design.

An important aspect of Human-Robot Interaction research is the conviction that user evaluation is the only way to assess such interaction. As Nielsen (1993) asserts: "User testing with real users is the most fundamental usability method and is in some sense irreplaceable, since it provides direct information about how people use computers and what their exact problems are with the concrete interface being tested".

\footnotetext{
1 This work has been partially supported by the Spanish Ministry of Science and Innovation under the project ARABOT -DPI2010-21247-C02-01 and by the Comunidad de Madrid funding program
}

In our iterative design process of the PDA interface, the first version underwent user testing, the results of which were used to inform the next version of the interface. In addition to exploring the design of the interface, we also adapted the robotic system in order to make it fit the requirements of the interaction. In this paper, we will discuss the design of the first version of our graphical user interface (GUI) and interaction system. The major effort of the first version was to provide the operator with the situational awareness required to tele-operate a robot in order to explore an unknown environment. We will first review the bibliography currently existing on the topic. Next, we will lay out the design and evaluation of the first version of our interface prototype. The evaluation sets the stage for the second prototype, which will be described at the end of the paper. This second prototype focuses on issues surrounding an enhanced operator situational awareness and operation modalities. New operation modalities and some usability modifications to increase operator situational awareness will emerge.

\section{BACKGROUND AND MOTIVE STATEMENT}

The commonly accepted SA definition was given by Endsley (1988) and adapted to HRI by Yanco et al. (2003) as "the understanding that the human has of the location, activities, status, and surroundings of the robot; and the knowledge that the robot has of the human's commands necessary to direct its activities and the constraints under which it must operate". Yanco et al. (2003) distinguishes three components within the concept of SA: human-robot SA, robot-human SA, and the human's overall mission awareness. Within the humanrobot awareness two aspects are important for the purposes of this paper: location awareness, defined as a map-based concept, allowing the user to locate the robot in the scenario, and surroundings awareness pertaining to obstacle avoidance, allowing the user to recognize the immediate surroundings of the robot (Drury et al. (2007)).

In order to better understand how SA enhances the operator's performance when he is driving a robot is useful to introduce two important concepts from human spatial-cognition: route knowledge and survey knowledge. The distinction be- 
tween route and survey knowledge helps in identifying and understanding the cognitive skills required by a human operator remotely controlling a robot. The route perspective is closely linked to perceptual experience: it occurs under the egocentric perspective in a "retinomorphous reference system", that is, one is able to perceive himself in the space (Herrmann (1996)), with a special emphasis on spatial relations between objects composing the scene an agent is situated in. This is for example the case of an operator driving a robot with a tridimensional perspective on a screen, simulating the visual information that he or she would obtain by directly navigating in that environment. Examples can be seen in Valero et al. (2010); Nielsen and Goodrich (2006); Driewer et al. (2008). The problem is that such information integration cannot be currently implemented in PDAs due to their reduced size and computation capabilities. Route-based information, from a ground perspective, is stored in memory to keep trace of turning points, distances and landmarks or relevant points of reference in the observed context. In contrast, survey perspective is characterized by an external and allocentric perspective, such as an aerial or maplike view, allowing direct access to the global spatial layout (Cohen (1989)) as it would be if the operator had a device by which he or she can have a global, aerial view of the robot and its environment. Previous studies have shown that a navigator having access to both perspectives exhibits more accurate performances (Herrmann (1996)).

We can appreciate a relation between location awareness and survey knowledge, while surroundings awareness relates to route knowledge. Our case study consists of a human operator driving remotely a robot using a human-robot interface. When the operator is not physically in the navigation scenario, the interface must enhance his or her spatial cognitive abilities by offering multilevel information about the environment (route and survey). Complex interfaces can provide different perspectives of the environment (bird's eye view or first-person view). Such information allows an operator looking at a GUI to have more than one perspective at the same time. Contrarily, if the operator is in the scenario, part of the information can be acquired by direct observation, depending on the visibility conditions. In such situations less information is required in the GUI. The goal of our PDA interface is to take benefit of the possibility of being in the scenario that provides carrying a hand-held device.

These spatial-cognitive aspects should be taken in consideration when designing a human-robot interface for remote teleoperation. According to them, and considering a GUI that can be carried in a hand-held device, we can say that intra-scenario operator mobility is a great advantage in the context of acquiring situational awareness in robot tele-operation, as the operator has visual access to the environment and in some situations may have visual contact with the robot.

In search and exploration missions, such as disaster situations or scheduled operations, a human team may be composed of on-site operators, who can carry only hand-held devices, and remote operators, who have access to wider computerized systems. Even if remote operators, using powerful work stations, can visualize and process a larger amount of data, responders carrying a PDA interface can boost the pervasiveness of robotic systems in mobile applications where operators cannot be pinned down in a particular place. Even if mobile devices are less powerful than desktop computers, they offer the operator the capacity to move, thus allowing him partially to view the actual scenario and the robot that he is controlling. The disadvantages related to device limitations could be balanced by the advantage of mobility. Mobility could facilitate better situational awareness and so enhance the control of the robot. First responders could control a robot team with a PDA interface while having a partial view of the environment, and thus could obtain on-field information not retrievable by the robot's sensors. In a case such as the Chernobyl disaster, first responders could control a robot team with a PDA interface while having a partial view of the environment, and thus obtain onfield information not retrievable by the robot sensors. The PDA interface is obviously suited for exploiting just this advantage of mobility.

With these sorts of goals in mind, some research groups have designed graphical user interfaces for PDAs. In the last few years, many interfaces have been developed for use on handheld devices: for military applications: Fong et al. (2001, 2003b,a); for exploration and navigation: Kaymaz-Keskinpala and Adams (2004); Kaymaz-Keskinpala et al. (2003); Hedström et al. (2005); and for service robots: Perzanowski et al. (2001); Httenrauch and Norman (2001); Skubic et al. (2003).

With all this background we designed the first interface prototype, which is presented in the following section.

\section{PDA INTERFACE}

In this section we will describe our PDA interface.

\subsection{Operator Displays}

Due to the reduced size of a PDA and its computational limitations, the display cannot present on-screen all the data provided by the robot sensors. In order to preserve the same functions offered by most desktop-based interfaces (laser and sonar readings, and the enviroment's map), we implemented them using various simplified layouts. This underlines how critically important it is to present the operator only with the crucial data, as each layout change implies a longer interaction time with the device. Another critical point was to consider the slower input capacities of the operator with a PDA: by means of a touch screen and a four-way navigation joystick. Thus, it is important to minimize the number of interactive steps required to change a setting or to command the robot.

The PDA has two kinds of 2D views, each selectable with its own tab. The first, centred in the robot, is the Laser View (Figure 1(a)). The second is the Map View (Figure 1(b)).

A third tab (Figure 1(c)) is dedicated to the Robot Control functionalities.

Laser View. This is an egocentric view attached to the robot; it remains stationary at the bottom part of the display. It offers a precise real-time local representation of what obstacles the robot is facing. The graphic for this view is very simple and no other information relative to the robots status (orientation, speed, etc.) is provided. This view can be zoomed in and out.

Map View. This is an allocentric view relative to the explored environment. It allows the operator to retrieve the map of the explored area. It can be zoomed in or out. By clicking on a point within the map, the operator commands the robot to go to a target point (shared control). As the whole map is computationally expensive, we decided to eliminate periodic self-updating; the map refreshes itself only on the users demand. 


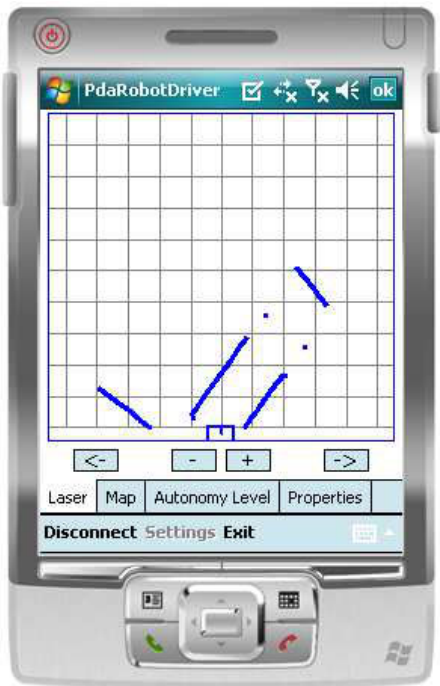

(a) PDA Interface v.1: Laser View

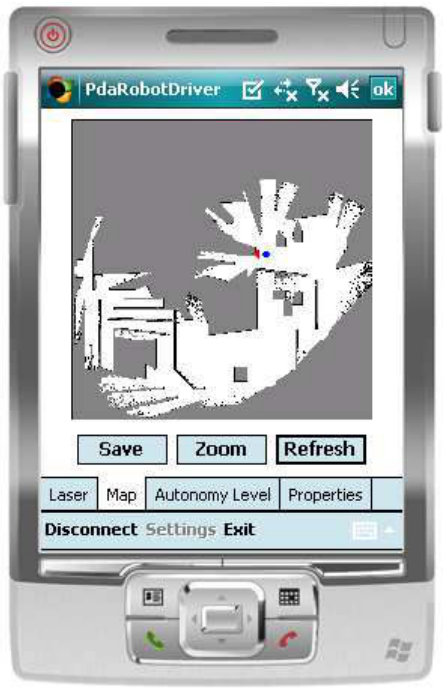

(b) PDA Interface v.1: Map View

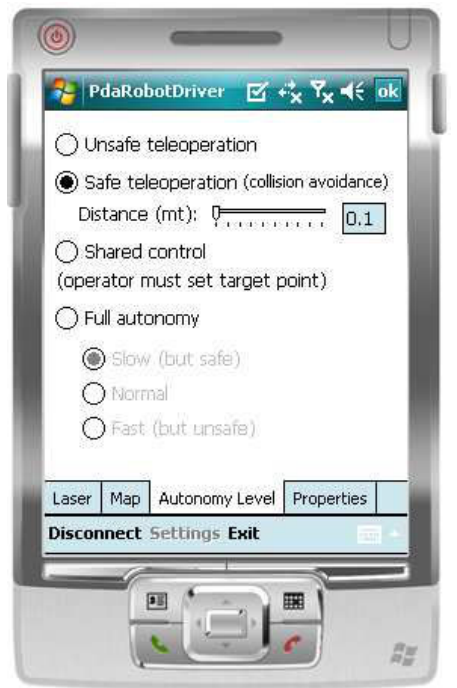

(c) PDA Interface v.1: Autonomy Levels

Fig. 1. PDA Interface.

Autonomy Levels Panel. This allows the user to set his desired robot control mode. In Shared Control or Autonomy Mode.

\subsection{Operation Modalities}

When the operator is in the laser view or the map view mode, he can tele-operate the robot using the cursor of the PDA. There are two speed control modalities:

- Fixed control. When the operator presses the "up" cursor, the robot moves forward at a fixed speed, which is the same for right, left and back directions. When the operator releases the cursor, the robot stops.

- Incremental control. Each time the operator presses the cursor, the robot speed increases or decreases by increments or decrements (according to the cursor). To stop the robot, the operator must press the middle button.

For Shared Control, the operator must select the map view. The operator can use the pen-stick to click onto a desired target point the robot is then supposed to reach autonomously.

\section{INTERFACE EVALUATION}

The evaluation of the first prototype was designed to measure the usability and performance of the interface developed.

\subsection{Experiment Design and Procedure}

Students were enrolled and a disaster scenario was simulated on the playground of the University. The experiments involved twenty-four subjects, nineteen undergraduates and five $\mathrm{PhD}$ candidates ranging in age between 20 and 30 and distributed among four females and twenty males. None of the participants had previous experience with the interface. Every subject went through a twenty-minute training program to acquire a basic knowledge of the functionalities provided by the interface. After the training they ran through the experiment. Each subject had a single trial.

Experiments were conducted with a Pioneer 2AT robot in both indoor and outdoor scenarios. Subjects were asked to explore a maze and navigate along a 15 meters long path with narrow spaces and clustered areas. The subjects could see the outdoor scenario and the robot but not the indoor scenario.

We asked users to "think aloud" during the task, as we wished afterwards to apply the LASSO evaluation technique described by Drury et al. (2007).

We also compared the performance of the operators using the PDA interface wrt. the operators making the same task using our Desktop-based interface, described in Valero et al. (2010). Twelve students drove the robot using the PDA and twelve using a Desktop computer. None of the operators could see the robot nor the scenario in which the robot was moving. In the following we will show the performance results and the usability analysis resulting from the LASSO evaluation.

\subsection{Results}

Performance We measured the area that the robot covered, and compared the area covered when the operators used the Desktop-based interface wrt. the area covered when using the PDA. The independent variable was the Interface Type $\in\{P D A$ interface, desktop interface $\}$, while the rest of the factors remained unchanged. The dependent variable was the covered area by the robot in square meters. An area was considered covered if it had been mapped. Results can be seen in Figure 2. A direct observation of the areas explored by the operator using the desktop and the operator using the PDA reveal that the former performs considerably better. The analysis showed a significant interaction between Interface and Time $[F(19,361)=$ 13.65, $p<.00001]$. A planned comparison for each level of time was calculated, indicating that at minute 1.5 of exploration the difference between Desktop and PDA, in terms of explored area, is just significant $[p<.05]$. Then it remains significant and grows at each level of Time

We then analyzed the time operators needed to complete the three different scenarios in which the robot should navigate: maze, cluttered area and narrow corridor.The independent variable was the Interface Type. As for the dependent variable, we were interested in the Navigation Time, measured in seconds. 


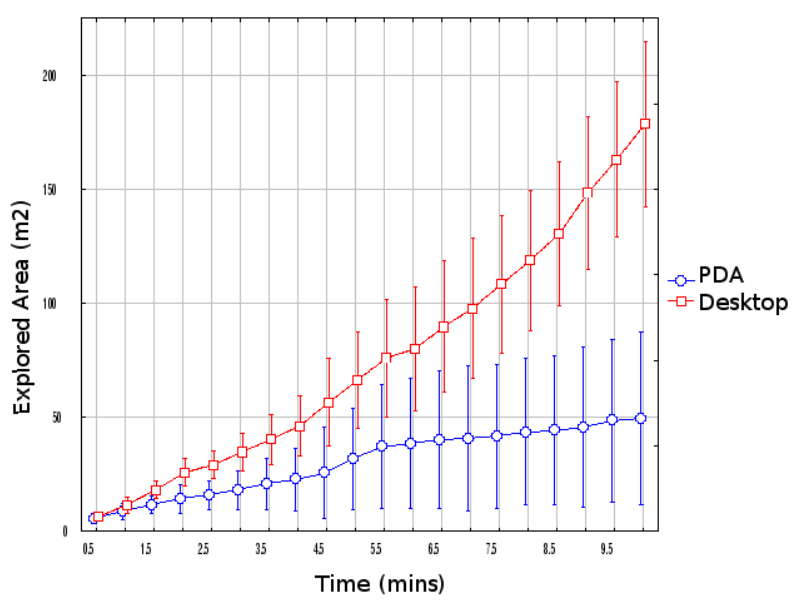

Fig. 2. Covered area in square meters by the operator using the PDA (bottom curve) and the operator using the desktop interface

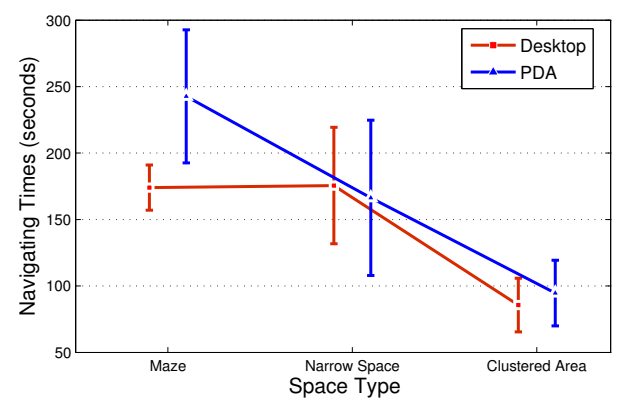

Fig. 3. Operator using PDA wrt. operator using desktop. Mean times and $95 \%$ Bonferroni confidence intervals are represented

Results can be seen in Figure 3. The first ANOVA on navigation times resulted non significant $[F<1]$ revealing no difference among the driving times between interfaces in the cluttered area and narrow spaces, while there was significant difference for the maze space.

The performance of the operators using both interfaces are practically identical when they are only dealing with obstable avoidance and motion control, that must be performed in the narrow and cluttered spaces. In this type of spaces, surroundings awareness is required for avoiding collisions and navigating, while location awareness is not required. Conversely, when they must explore an area, and thus be able to localize themselves inside the scenario (maze and exploration task) the performance of the operators using the PDA is smaller than those using the desktop-based interface (Figures 2 and 3). We can conclude from here that the weakest point of the PDA interface relates with the location awareness. These results were contrasted with the usability analysis shown in the following.

Usability Analysis We enumerate here the chief usability concerns on which the majority of subjects agreed. We will list the positive and negative usability remarks regarding the operators situational awareness.

\section{Surroundings Awareness}

Negative Remarks
- Laser View. As the robot is indicated at the bottom of the screen (see Figure 1(a)) it is difficult to keep track of the obstacles behind the robot.

- Map View. Too small to provide proper surroundings awareness. The use of an arrow to indicate robot orientation (see Figure 1(b)) hides part of the map.

\section{Positive Remarks}

- Laser View. The position of obstacles in front of the robot is quite precise and is sufficient for navigating even in narrow spaces.

- Map View. In open spaces, this view is sufficient for navigating without colliding with obstacles.

\section{Location Awareness}

\section{Negative Remarks}

- Laser View

- It is very difficult to keep track of the position and orientation of the robot.

- It is very difficult to know what places the robot has visited earlier.

\section{- Map View}

- It is easy to forget the path the robot has followed.

The map design is very small.

\section{Positive Remarks}

- Laser View. This view was inadequate for providing location awareness and thus there was no positive remark.

- Map View. The view is adequate for knowing were the robot is.

Status and Activity Awareness As for robot status and activities, the operators would have liked to know the speed of the robot (at least to know whether the robot was moving or still), which was not indicated by the interface. This was an issue raised by all the subjects.

One of the major weaknesses in the operation of the robot was the size of the cursor pad. On a standard PDA device, the cursor is quite small and not designed to be used continuously and precisely. Almost all the subjects wished to have another input device. Most operators preferred the tele-operation modality in which they could increase and decrease the speed and were unfavourable to the modality with a set speed. They argued that, while the fixed speed can be very convenient (for example, when the operator releases the cursor, the robot stops), there are areas calling for greater speed-such as open spaces-as well as areas calling for greater slowness — such as narrow spaces.

In conclusion, for the PDA interface, the surroundings awareness is provided mainly by the laser view, while the contribution of the map view is negligible. Conversely, the laser view is not appropriate for location awareness and even leads to confusion. The major problem with this interface is that the operator must change view modes in order to acquire a proper location and surrounding awareness. Since such view shifts are timeconsuming due to the reduced computation capabilities of the PDA, the operator tends to use only one of the views provided, even though no single view is sufficient for the task at hand.

Another problem with the PDA interface lies in its low computation speed and its communications latency. Given both of these issues, the refresh rate of the displayed data is too high to be compatible with fast and safe operation. A related problem is 
that it is difficult to tell whether the robot is not moving or if the problem is simply that the display has not yet been refreshed.

\subsection{Usability Heuristics}

We summarize the results of the experiments based on Nielsen's heuristics. The results can be seen in the following table.

\begin{tabular}{|l|l|}
\hline Heuristic & PDA \\
\hline $\begin{array}{l}\text { Required information should be } \\
\text { present and clear }\end{array}$ & $\begin{array}{l}\text { Rear obstacles should be visible. } \\
\text { Map is too small. }\end{array}$ \\
\hline $\begin{array}{l}\text { Prevent errors if possible, if not, } \\
\text { help users diagnose and recover }\end{array}$ & $\begin{array}{l}\text { Very difficult to distinguish robot } \\
\text { errors from device limitations }\end{array}$ \\
\hline $\begin{array}{l}\text { Use metaphors and language the } \\
\text { users already know }\end{array}$ & Yes \\
\hline Make it efficient to use & $\begin{array}{l}\text { The device is too limited and effi- } \\
\text { ciency is low }\end{array}$ \\
\hline $\begin{array}{l}\text { Design should be aesthetic and min- } \\
\text { imalist }\end{array}$ & $\begin{array}{l}\text { Yes, but the window for choosing } \\
\text { the autonomy level should be inte- } \\
\text { grated with the others. }\end{array}$ \\
\hline $\begin{array}{l}\text { Make the architecture scalable and } \\
\text { support evolution of platforms }\end{array}$ & $\begin{array}{l}\text { This is difficult due to the device } \\
\text { limitations }\end{array}$ \\
\hline Simplify tasks through autonomy & Yes \\
\hline Allow precise control & $\begin{array}{l}\text { Yes, but cursor pad is too small and } \\
\text { difficult to use with precision }\end{array}$ \\
\hline
\end{tabular}

Table 1: Adapted Nielsen Heuristics

\section{INTERFACE EVOLUTION}

The evaluation presented above propelled the evolution of the interface toward the next prototype, version 2 . The limitations of the PDA do not allow the implementation of all the user's demands. Nevertheless, we developed a new prototype, the second version, which introduces important improvements over the first prototype. This version is shown in Figures 4(a) and 4(b).

The innovations are:

- Laser/Sonar View. Figure 4(a).

- The robot has been moved to the middle of the screen, and the sonar readings (including rear sonars) are also included in the display.

- Ray-tracing has been applied, in order to distinguish free areas from unknown areas.

- The operator can also change the zoom of this view.

- The operator can control the robot speed by touching the screen directly. Transparent cursors avoid hiding information. The operator can click on the direction that he wants the robot to take, and the speed (linear speed and jog) is set according to that direction. If the operator clicks on the robot, the robot stops. This works in both speed control modalities: incremental and pre-set.

- The robot is drawn with solid color when is moving, and only the contour when it is still.

- A menu has been added to enable the selection of the operation mode, so that the operator does not need to change from one display to another.

- Map View. Figure 4(b).

- The operator can select an area on the map view to be zoomed.

- The control of the speed and jog is also integrated into the screen.

- A menu for changing the autonomy mode is also present on the screen.

\section{CONCLUSIONS}

In this paper, we have presented the first and second prototypes of our human-robot interface for PDA devices. The first prototype was designed taking account of the most important guidelines present in the literature. Previous interface designs were reviewed and their experimental evaluation was taken as a basis for our interface development.

The main contributions presented in this paper are:

(1) Usability analysis of a PDA human-robot interface for exploration robotics.

(2) Set of principles for a robotic interface design that would provide the operator with both surroundings and location awareness. The GUI provides different views, permitting the operator to use the most suitable according to the task.

(3) Design, implementation and evaluation of a PDA GUI prototype. The GUI was considered by the users as simple and minimalist. The information is clearly presented on the screen on an intuitive way and following the user requirements.

(4) After conducting user evaluation, a second prototype was designed and implemented according to the lessons learnt.

Considering the real application scenario of such an iterface, we can imagine a team of operators, with some of them working remotely, using desktop-based devices, while others stay closer to the operating scenario, thanks to the PDA device. The coordination of such team of operators can provide a better sytem performance, as under some circumstances remote operators can exploit better the robot navigation capabilities, while under other conditions the operator carrying the PDA can have an advantageous situation to control the robot. In Valero et al. (2009a) and Valero et al. (2009b), on the basis of extensive performance experimentation comparing our desktop-based interface wrt. the PDA-based interface, we provide support (and also some confutation) of this claim. The experiments presented in those papers were performed in order to identify the most suitable operator interface for controlling a mobile robot depending on the task and mobility/visibility of the operator.

\section{REFERENCES}

Adams, J.A. (2002). Critical considerations for human-robot interface development. Technical report, 2002 AAAI Fall Symposium: Human Robot Interactiomn Technical Report. Cohen, G. (1989). Memory in the real world. Hove: Erlbaum.

Driewer, F., Sauer, M., and Schilling, K. (2008). Design and evaluation of an user interface for the coordination of a group of mobile robots. In 17th Iternational Symposium on Robot and Human Interactive Communication. RO-MAN 2008, 237-242.

Drury, J.L., Hestand, D., Yanco, H.A., and Scholtz, J. (2004a). Design guidelines for improved human-robot interaction. In Extended abstracts of the 2004 Conference on Human Factors in Computing Systems, 1540.

Drury, J.L., Keyes, B., and Yanco, H.A. (2007). Lassoing hri: analyzing situation awareness in map-centric and videocentric interfaces. In Proceedings of the Second ACM SIGCHI/SIGART Conference on Human-Robot Interaction, $279-286$.

Drury, J.L., Yanco, H.A., and Scholtz, J.C. (2004b). Beyond usability evaluation: Analysis of human-robot interaction at a major robotics competition. Human-Computer Interaction Journal. 


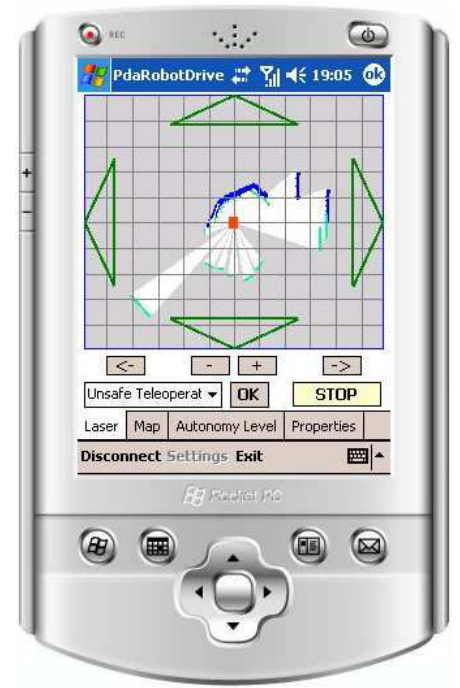

(a) Laser/Sonar View

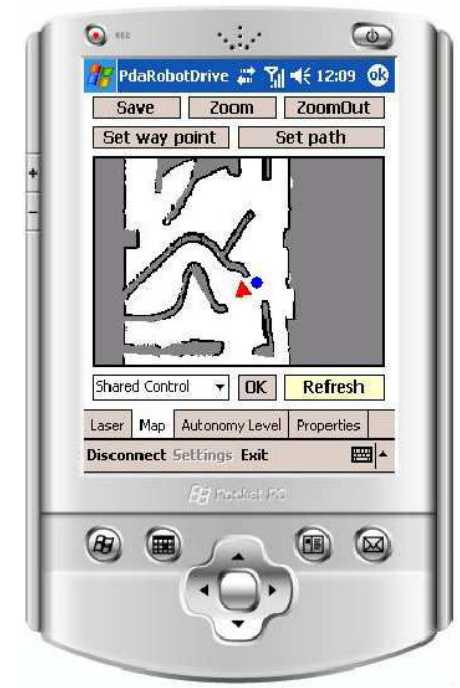

(b) Map View

Fig. 4. PDA Interface v.2.

Endsley, M.R. (1988). Design and evaluation for situation awareness enhancement. In Proceedings of the Human Factors Society 32nd Annual Meeting, 97-101.

Fong, T., Thorpe, C., and Glass, B. (2003a). Pdadriver: A handheld system for remote driving. In In IEEE International Conference on Advanced Robotics.

Fong, T., Thorpe, C.E., and Baur, C. (2001). Advanced interfaces for vehicle teleoperation: Collaborative control, sensor fusion displays, and remote driving tools. Autonomous Robots, 11(1), 77-85.

Fong, T., Thorpe, C.E., and Baur, C. (2003b). Robot, asker of questions. Robotics and Autonomous Systems, 42(3-4), 235243.

Hedström, A., Christensen, H.I., and Lundberg, C. (2005). A wearable gui for field robots. In Field and Service Robotics, 367-376.

Herrmann, T. (1996). Blickpunkte und blickpunktsequenzen. In Sprache \& Kognition, volume 15 of 217-233.

Humphrey, C.M., Henk, C., Sewell, G., Williams, B.W., and Adams, J.A. (2007). Assessing the scalability of a multiple robot interface. In Proceedings of the Second ACM SIGCHI/SIGART Conference on Human-Robot Interaction, HRI 2007, Arlington, Virginia, USA, March 10-12, 2007, 239-246.

Httenrauch, H. and Norman, M. (2001). Pocketcero - mobile interfaces for service robots. In In Proceedings of the International Workshop on Human Computer Interaction with Mobile Devices.

Kaymaz-Keskinpala, H. and Adams, J.A. (2004). Objective data analysis for a pda-based human robotic interface. In Proceedings of the IEEE International Conference on Systems, Man \& Cybernetics, 2809-2814.

Kaymaz-Keskinpala, H., Kawamura, K., and Adams, J.A. (2003). Pda-based human-robotic interface. In Proceedings of the IEEE International Conference on Systems, Man \& Cybernetics, volume 4, 3931 - 3936.

Nielsen, C.W. and Goodrich, M.A. (2006). Comparing the usefulness of video and map information in navigation tasks. In Proceedings of the 1st ACM SIGCHI/SIGART Conference on Human-Robot Interaction, HRI 2006, 95-101.

Nielsen, J. (1993). Usability Engineering. Academic Press.

Perzanowski, D., Schultz, A.C., Adams, W., Marsh, E., and Bugajska, M.D. (2001). Building a multimodal human-robot interface. IEEE Intelligent Systems, 16(1), 16-21.

Scholtz, J., Young, J., Drury, J.L., and Yanco, H.A. (2004). Evaluation of human-robot interaction awareness in search and rescue. In Robotics and Automation, 2004. Proceedings ICRA'04, volume 3, 2327 - 2332. IEEE.

Skubic, M., Bailey, C., and Chronis, G. (2003). A Sketch Interface for Mobile Robots. In In Proc. IEEE 2003 Conf. on SMC, 918-924.

Valero, A., de la Puente, P., Rodriguez-Losada, D., and Matia, F. (2010). Evaluating a human-robot interface for exploration missions. In (IFAC 7th Symposium on Intelligent Autonomous Vehicles. IFAC.

Valero, A., Randelli, G., Saracini, C., Botta, F., and Mecella, M. (2009a). The advantage of mobility, mobile tele-operation for mobile robots. In Proceedings of the AISB-HRI Symposium "New Frontiers in Human - Robot Interaction.

Valero, A., Randelli, G., Saracini, C., Botta, F., and Nardi, D. (2009b). Give me the control, i can see the robot! In Safety, Security Rescue Robotics (SSRR), 2009 IEEE International Workshop on, 1 -6. doi:10.1109/SSRR.2009.5424157.

Yanco, H.A. and Drury, J.L. (2007). Rescuing interfaces: A multi-year study of human-robot interaction at the aaai robot rescue competition. Auton. Robots, 22(4), 333-352.

Yanco, H.A., Drury, J.L., and Scholtz, J. (2003). Awareness in human-robot interactions. In Proceedings of the IEEE Conference on Systems, Man and Cybernetics, Washington, DC, October 2003. 